IJMMS 31:10 (2002) 603-609

PII. S0161171202106041

http://ijmms.hindawi.com

(c) Hindawi Publishing Corp.

\title{
SUR LES FONCTIONS SÉPARÉMENT EXCESSIVES
}

\author{
MOHAMED EL KADIRI
}

Reçu le 7 juillet 2001 et sons forme réviseé le 28 décembre 2001

On considère deux résolvantes basiques sur deux espaces mesurables $(E, \mathscr{E})$ et $(F, \mathscr{F})$ associées à des semi-groupes de noyaux $\left(P_{t}\right)$ et $\left(Q_{t}\right)$. On montre que si $E$ et $F$ sont radoniens, alors toute fonction sur $E \times F$ séparément excessive pour les semi-groupes $\left(P_{t}\right)$ et $\left(Q_{t}\right)$ est excessive pour le semi-groupe $\left(P_{t} \otimes Q_{t}\right)$ (ou sa résolvante), ce qui généralise un résultat bien connu de Cairoli (1967).

2000 Mathematics Subject Classification: 60J35, 60J45.

1. Introduction. Soient $\left(P_{t}\right)$ et $\left(Q_{t}\right)$ deux semi-groupes de noyaux (sous-markoviens) sur des espaces mesurables $(E, \mathscr{E})$ et $(F, \mathscr{F})$ respectivement. Le produit tensoriel de ces deux semi-groupes, noté par $\left(P_{t} \otimes Q_{t}\right)$, est défini sur l'espace mesuré $(E \times F, \mathscr{E} \otimes \mathscr{F})$ par

$$
P_{t} \otimes Q_{t}((x, y), A \times B)=P_{t}(x, A) Q_{t}(y, B)
$$

pour tout couple $(x, y) \in E \times F$ et tous $A \in \mathscr{E}, B \in \mathscr{F}$. On a alors

$$
\left(P_{t} \otimes Q_{t}\right)(f \otimes g)=P_{t}(f) Q_{t}(g)
$$

pour tout couple $(f, g)$ de fonctions $f \mathscr{E}$-mesurable $\geq 0$ et $g \mathscr{F}$-mesurable $\geq 0$.

Il est facile de vérifier que $\left(P_{t} \otimes Q_{t}\right)$ est un semi-groupe de noyaux sur $(E \times F, \mathscr{E} \otimes \mathscr{F})$.

On dit qu'une fonction $f$ sur $E \times F$ à valeurs dans $\mathbb{\mathbb { R }}_{+}$est séparément excessive si, pour tout couple $(x, y) \in E \times F$, les fonctions $f(\cdot, y)$ et $f(x, \cdot)$ sont respectivement excessives pour les semi-groupes $\left(P_{t}\right)$ et $\left(Q_{t}\right)$. Une question intéressante est de savoir si une telle fonction est excessive pour le semi-groupe $\left(P_{t} \otimes Q_{t}\right)$.

Si $E$ et $F$ sont des espaces localement compacts, $\mathscr{E}$ et $\mathscr{F}$ leur tribus boréliennes respectives et si les semi-groupes $\left(P_{t}\right)$ et $\left(Q_{t}\right)$ sont fortement fellériens, Cairoli a montré dans [2] que la réponse à la question précédente est positive.

Si $E$ et $F$ sont des ouverts fins de $\mathbb{R}^{m}$ et $\mathbb{R}^{n}$ respectivement, nous avons montré dans [4], en nous inspirant de la méthode de Cairoli, qu'une fonction séparément finement surharmonique localement bornée inférieurement sur $U \times V$ est finement surharmonique. À ce sujet, de La Pradelle nous a indiqué dans une communication personelle que notre méthode peut s'appliquer au cadre plus général des résolvantes basiques. Plus précisemment, on a le théorème suivant :

THÉORÈme 1.1. Soient $(E, \mathscr{E})$ et $\left(F, F_{F}\right)$ deux espaces mesurables, $\left(P_{t}\right)$ et $\left(Q_{t}\right)$ deux semi-groupes dont les résolvantes sont basiques. Si E et $F$ sont radoniens, alors toute fonction séparément excessive sur $E \times F$ est excessive pour le semi-groupe $\left(P_{t} \otimes Q_{t}\right)$. 
La méthode de Cairoli des noyaux et résolvantes fortement fellériens [2] ne s'applique pas directement, nous allons l'adapter en utilisant des compactifications de Ray associées aux résolvantes basiques. Nous allons en fait montrer que toute fonction séparément excessive est universellement mesurable, ce qui constitue la clef du problème.

Le théorème 1.1 contient le résultat de Cairoli puisque, dans le cas des semi-groupes fortement fellériens, les fonctions excessives sont s.c.i. (semi-continue inférieurement) et donc les résolvantes associées sont basiques d'après [3, chapitre XII, Section 41].

Dans tout ce travail, les semi-groupes et les résolvantes considérés seront supposés sous-markoviens.

2. Rappels sur les résolvantes basiques. Pour les notions de résolvantes et de semi-groupes de noyaux nous renvoyons au livre de Dellacherie et Meyer [3].

Une famille résolvante $\left(V_{\lambda}\right)$ de noyaux sur un espace mesurable $(E, \mathscr{E})$ est dite basique ou absolument continue s'il existe une mesure positive $m$ bornée sur $(E, \mathscr{E})$ telle que, pour tout $x \in E$ et tout $\lambda>0$, la mesure $V_{\lambda}(x, d y)$ est absolument continue par rapport à $m$. Une telle mesure sera appelée mesure de base de la résolvante $\left(V_{\lambda}\right)$.

On dit qu'un semi-groupe $\left(P_{t}\right)$ sur $(E, \mathscr{E})$ est basique s'il existe une mesure positive $m$ bornée sur $(E, \mathscr{E})$ telle que, pour tout $t>0$ et tout $x \in E$, la mesure $P_{t}(x, d y)$ est absolument continue par rapport à $m$. La mesure $m$ est alors appelée mesure de base du semi-groupe $\left(P_{t}\right)$.

Un noyau $V$ sur $(E, \mathscr{E})$ est dit basique s'il existe une mesure $m$ positive bornée sur $(E, \mathscr{E})$ telle que pour tout $x \in E$, la mesure $V(x, d y)$ est absolument continue par rapport à $m$. La mesure est appelée mesure de base de $V$. Il est clair qu'une résolvante est basique de base $m$ si et seulement si son noyau potentiel $V$ est basique de base $m$. De même, une famille résolvante de noyaux associée à un semi-groupe $\left(P_{t}\right)$ sur $(E, \mathscr{E})$ est basique si $\left(P_{t}\right)$ est basique ; ils ont alors une même mesure de base. On remarquera toutefois, d'après (voir [3, pages 107-108]), que la réciproque de ce dernier résultat n'est pas toujours vraie.

Lorsque la résolvante $\left(V_{\lambda}\right)$ est basique et sépare les points de $E$, on peut définir une topologie métrisable séparable sur $E$ et une résolvante $\left(\bar{V}_{\lambda}\right)$ sur $E$ admettant les mêmes fonctions excessives que $\left(V_{\lambda}\right)$, telles que, pour toute fonction $\mathscr{E}$-mesurable bornée $f \geq 0$ sur $E$ et tout $\lambda>0$, la fonction $\bar{V}_{\lambda} f$ est continue sur $E$ (voir [3, Section 90, page 77]). Cette topologie provient d'une compactification de Ray $\bar{E}$ de $E$. La résolvante $\left(\bar{V}_{\lambda}\right)$ est construite à partir du noyau $\bar{V}$ défini par

$$
\bar{V} f=V(a f)
$$

pour toute fonction mesurable $f \geq 0$, où $a$ est une fonction mesurable $>0$ bornée convenablement choisie. De plus, la tribu de Borel de $E$ est contenue dans $\mathscr{E}$ (voir construction de $\bar{E}$ dans [3, Section 87, p. 73]).

Soient $(E, \mathscr{E})$ et $(F, \mathscr{F})$ deux espaces mesurables et soient $\left(U_{\lambda}\right)$ et $\left(V_{\lambda}\right)$ deux résolvantes basiques sur $(E, \mathscr{E})$ et $(F, \mathscr{F})$ de bases $m_{E}$ et $m_{F}$ respectivement. On note par $\bar{E}$ et $\bar{F}$ les compactifiés de Ray de $E$ et $F$ pour les résolvantes $\left(U_{\lambda}\right)$ et $\left(V_{\lambda}\right)$ respectivement. On suppose que $E$ et $F$ sont radoniens, c'est-à-dire plongeables dans des espaces com- 
pacts métrisables séparables en tant que parties universellement mesurables (voir [3, p. 75]). On rappelle que $E$ et $F$ sont alors, grâce au théorème de Lusin, des parties universellement mesurables de $\bar{E}$ et $\bar{F}$ respectivement, ce qui permet d'écrire $E \times F$, pour toute mesure positive bornée $\tau$ sur $E \times F$, sous la forme $E \times F=\bigcup_{m, n} H_{m} \times K_{n} \cup N$, où $\left(H_{n}\right)$ (resp., $\left.\left(K_{n}\right)\right)$ est une suite de compacts de $\bar{E}$ (resp., $\bar{F}$ ) et $N$ est $\tau$ négligeable, ce qui est essentiel pour démontrer qu'une fonction séparément excessive est universellement mesurable.

Nous dirons qu'une fonction sur $E \times F$ est séparément mesurable si pour tout $x \in E$ (resp., $y \in F$ ) la fonction $f(x, \cdot)$ (resp., $f(\cdot, y)$ ) est $\mathscr{F}$-mesurable (resp., $\mathscr{F}$ )-mesurable.

THÉORÈme 2.1. Soient $\lambda, \mu>0$ et $f$ une fonction réelle bornée sur $E \times F$ séparément mesurable telle que l'intégrale itérée

$$
g(x, y)=\int \bar{U}_{\lambda}(x, d u) \int \bar{V}_{\mu}(y, d v) f(u, v)
$$

existe pour tout couple $(x, y) \in E \times F$. Alors la fonction $(x, y) \mapsto g(x, y)$ est universellement mesurable.

DÉmonstration. Soit $\tau$ une mesure positive bornée sur $E \times F$. Comme $E$ et $F$ sont des parties universellement mesurables de $\bar{E}$ et $\bar{F}$, on peut trouver deux suites $\left(H_{n}\right)$ et $\left(K_{n}\right)$ de parties compactes de $E$ et $F$ respectivement telles que $\tau\left(E \times F \backslash \bigcup_{m, n} H_{m} \times\right.$ $\left.K_{n}\right)=0$. Pour tout entier $m$ et tout $y \in F$, l'application partielle $x \mapsto g_{y}(x)=g(x, y)$ est continue sur $H_{m}$. Soit $n \in \mathbb{N}$, pour toute suite $\left(y_{i}\right)$ de points de $K_{n}$ convergente vers $y \in K_{n}$, on montre que la suite des fonctions $g_{y_{i}}$ converge uniformément vers $g_{y}$ sur $H_{m}$. On en déduira que la fonction $g$ est continue sur $H_{m} \times K_{n}$, le théorème en résultera. Notons par $h_{y}$ la fonction $u \mapsto \int \bar{V}_{\lambda}(y, d v) f(u, v)$, alors on a $\lim _{i \rightarrow \infty} h_{y_{i}}=$ $h_{y}$ puisque pour tout $u \in E$, la fonction $v \mapsto f(u, v)$ est $\mathscr{F}_{\text {-mesurable bornée. Posons }}$ $h_{i}^{\prime}=\inf _{j \geq i} h_{y_{j}}$ et $h_{i}^{\prime \prime}=\sup _{j \geq i} h_{y_{j}}$. On a $h_{i}^{\prime} \uparrow h_{y}$ et $h_{i}^{\prime \prime} \downarrow h_{y}$ et par conséquent $\bar{U}_{\lambda} h_{i}^{\prime} \uparrow$ $\bar{U}_{\lambda} h_{y}$ et $\bar{U}_{\lambda} h_{i}^{\prime \prime} \downarrow \bar{U}_{\lambda} h_{y}$. Les fonctions intervenant dans ces deux dernières limites étant continues sur le compact $H_{m}$, la convergence a donc lieu uniformément sur $H_{m}$ d'après le théorème de Dini. Les inégalités

$$
\bar{U}_{\lambda} h_{i}^{\prime} \leq \bar{U}_{\lambda} h_{y_{i}} \leq \bar{U}_{\lambda} h_{i}^{\prime \prime}
$$

entrainent finalement que $\bar{U}_{\lambda}\left(h_{y_{i}}\right)$ converge uniformément vers $\bar{U}_{\lambda}\left(h_{y}\right)$ sur $H_{m}$, autrement dit, la suite $\left(g_{y_{i}}\right)$ converge uniformément vers $g_{y}$ sur $H_{m}$, d'où le résultat.

COROLlaire 2.2. Si $f$ est une fonction séparément excessive sur $E \times F$, alors $f$ est universellement mesurable.

DÉmonstration. Supposons d'abord que $f$ est bornée. Il est clair que l'intégrale itérée

$$
\int \bar{U}_{\lambda}(x, d u) \int \bar{V}_{\mu}(y, d v) f(u, v)
$$

existe pour tout couple $(x, y) \in E \times F$ puisque la deuxième intégrale est une fonction s.c.i. en $u$ (ce qui résulte du théorème de Fubini et de ce que les fonctions excessives de la résolvante $\left(U_{\lambda}\right)$ sont s.c.i.). D’après le théorème 2.1 la fonction $g$, définie par

$$
g(x, y)=\lambda \mu \int \bar{U}_{\lambda}(x, d u) \int \bar{V}_{\mu}(y, d v) f(u, v),
$$


est universellement mesurable. En faisant tendre $\lambda$ puis $\mu$ vers $+\infty$, on obtient que $f$ est universellement mesurable. Le cas général s'obtient en considérant les fonctions $f_{n}=\min (f, n)$.

3. Démonstration du théorème 1.1. Soit $\left(R_{t}\right)$ un semi-groupe basique sur un espace mesurable $(G, \mathscr{G})$. On dit qu'une fonction universellement mesurable $f \geq 0$ sur $G$ est surmédiane si $R_{t} f \leq f$ pour tout $t>0$. Si $f$ est surmédiane, la fonction $t \mapsto R_{t} f$ est décroissante. On dit qu'une fonction $\mathscr{G}$-mesurable $f \geq 0$ sur $G$ est excessive si elle est surmédiane et si $\lim _{t \rightarrow 0} R_{t} f=f$. La régularisée excessive d'une fonction surmédiane $f$, notée par $\hat{f}$, et définie par

$$
\hat{f}(x)=\lim _{t \rightarrow 0} R_{t} f(x), \quad \forall x \in G,
$$

est une fonction excessive. C'est la plus grande minorante excessive de $f$.

Proposition 3.1. Soit $h$ une fonction sur $E \times F$ séparément excessive pour le semigroupe $\left(P_{t} \otimes Q_{t}\right)$, alors $h$ est surmédiane.

DÉmonstration. On a vu que $h$ est universellement mesurable. Donc on a, d'après le théorème de Fubini,

$$
\begin{aligned}
P_{t} \otimes Q_{t}(h)(x, y) & =\iint h(u, v) P_{t}(x, d u) Q_{t}(y, d v) \\
& \leq \int h(x, v) Q_{t}(y, d v) \\
& \leq h(x, y)
\end{aligned}
$$

pour tout couple $(x, y) \in E \times F$.

DÉmonstration DU THÉORÈme 1.1. Supposons que les résolvantes $\left(U_{\lambda}\right)$ et $\left(V_{\lambda}\right)$ associées aux semi-groupes $\left(P_{t}\right)$ et $\left(Q_{t}\right)$ soient basiques de sorte que l'on puisse appliquer les résultats du deuxième paragraphe. Soit $f$ une fonction séparément excessive sur $E \times F$. D'après la proposition 3.1, $f$ est surmédiane pour le semi-groupe $\left(P_{t} \otimes Q_{t}\right)$. Soit $\widehat{f}$ la régularisée excessive de $f$, c'est-à-dire, la fonction définie par

$$
\hat{f}(x, y)=\lim _{t \rightarrow 0} P_{t} \otimes Q_{t} f(x, y),
$$

pour tout couple $(x, y) \in E \times F$, alors $\hat{f}$ est $\left(P_{t} \otimes Q_{t}\right)$-excessive. Nous allons prouver que $f=\widehat{f}$, ce qui démontrera le théorème. Supposons d'abord que $f$ soit bornée. Pour $(x, y) \in E \times F$ fixé, $\int P_{s}(x, d u) \int Q_{t}(y, d v) f(u, v)$ est une fonction décroissante de $s$ et de $t$, ce qui entraine que, d'après le théorème de convergence dominée,

$$
\lim _{t \rightarrow 0} P_{t} \otimes Q_{t} f(x, y)=\lim _{s \rightarrow 0} \int P_{s}(x, d u)\left(\lim _{t \rightarrow 0} \int P_{t}(y, d v) f(u, v)\right),
$$

d'où $\hat{f}(x, y)=f(x, y)$. Donc $f$ est $\left(P_{t} \otimes Q_{t}\right)$-excessive. Le cas général s'en déduit en considérant les fonctions $f_{n}=\min (f, n), n$ entier $\geq 1$. 
4. Applications. Soit $\left(P_{t}\right)$ le semi-groupe de la chaleur dans $\mathbb{R}^{d}, d \geq 3$, i.e., le semigroupe de transition de $\mathbb{R}^{d}$ donné par

$$
P_{t}(x, A)=\frac{1}{(\sqrt{2 \pi t})^{d}} \int_{A} \exp \left(-\frac{\|x-y\|^{2}}{2 t}\right) d y
$$

pour tout $x \in \mathbb{R}^{d}$ et toute partie borélienne $A$ de $\mathbb{R}^{d}$. Le processus de Markov associé à ce semi-groupe de transition est le mouvement brownien $B=\left(B_{t}\right)$ dans $\mathbb{R}^{d}$. Il est clair que le semi-groupe $\left(P_{t}\right)$ est basique de base la mesure de Lebesgue sur $\mathbb{R}^{d}$ (ou, si l' on veut une mesure bornée, la mesure de densité $1 /\left(1+x^{2}\right)$ par rapport à la mesure de Lebesgue).

Soit $U$ un domaine fin de $\mathbb{R}^{d}$ qu'on peut supposer régulier quitte à lui ajouter les points irréguliers de sa frontière fine $\partial_{f} U$, ce qui ne change pas le cône des fonctions finement surharmoniques $\geq 0$ en vertu du principe du prolongement par continuité fine car les points irréguliers de $\partial_{f} U$ forment un ensemble polaire. On note par $\tau_{U}$ le temps de sortie de $\left(B_{t}\right)$ de $U$. Alors $\tau_{U}$ est un temps terminal au sens de [1, définition 2.18] et donc $M=1_{\left[0, \tau_{U}\right.}$ [ est une fonctionnelle multiplicative pour le processus de Markov $\left(B_{t}\right)$. Le semi-groupe de transition $\left(P_{t}^{U}\right)$, subordonné à $\left(P_{t}\right)$, associé à $M$ est donné par

$$
\begin{aligned}
P_{t}^{U} f(x) & =E^{x}\left(f\left(B_{t}\right) M_{t}\right) \\
& =E^{x}\left(1_{\left[0, \tau_{U}[\right.} f\left(B_{t}\right) d t\right) .
\end{aligned}
$$

Notons par $\left(B_{t}^{U}\right)$ le processus de Markov à états dans $\mathbb{R}^{d}$ associé au semi-groupe $\left(P_{t}^{U}\right)$. Le processus $\left(B_{t}^{U}\right)$ est à trajectoires dans $U$.

Soit $\left(\mathscr{V}_{\lambda}\right)$ la famille résolvante sur $\mathbb{R}^{d}$ associée au semi-groupe $\left(P_{t}\right)$, c'est-à-dire, la résolvante définie par

$$
\mathscr{V}_{\lambda} f(x)=\int_{0}^{\infty} \exp (-\lambda t) P_{t} f(x) d t
$$

pour tout $\lambda>0$ et toute fonction borélienne $f \geq 0$ sur $\mathbb{R}^{d}$, et soit $\mathscr{V}$ le noyau potentiel de cette résolvante. Notons aussi par $\left(\mathscr{V}_{\lambda}^{U}\right)$ la résolvante, subordonnée à $\left(\mathscr{V}_{\lambda}\right)$, associée au semi-groupe $\left(P_{t}^{U}\right)$ et par $\mathscr{V}^{U}$ son noyau potentiel. La résolvante $\left(\mathscr{V}_{\lambda}^{U}\right)$ et le semigroupe $\left(P_{t}^{U}\right)$ sont basiques de base la restriction de la mesure de Lebesgue à $U$ ou une mesure équivalente. On note par $\mathscr{Y}(U)$ le cône des fonctions finement surharmoniques dans $U$. Moyennant un théorème d'approximation de Fuglède ([5, théorème 3]), on démontre le théorème suivant.

ThÉORÈme 4.1 [4, théorème 3.4]. Le cône $\mathscr{Y}(U)$ est identique au cône des fonctions excessives finies presque partout de la résolvante $\left(\mathcal{V}_{\lambda}^{U}\right)$ ou, ce qui revient au même, du semi-groupe $\left(P_{t}^{U}\right)$.

Soient $U$ et $V$ deux domaines fins de $\mathbb{R}^{m}$ et $\mathbb{R}^{n}$ respectivement, $m, n \geq 3$, alors $U \times V$ est ouvert fin de $\mathbb{R}^{m+n}$ et on a

$$
P_{t}^{U} \otimes P_{t}^{V}=P_{t}^{U \times V}
$$

En effet, on a $1_{\left[0, T_{U}[\right.} \cdot 1_{\left[0, T_{V}[\right.}=1_{\left[0, \tau_{U \times V}[\right.}$ et le produit tensoriel des semi-groupes de la chaleur dans $\mathbb{R}^{m}$ et $\mathbb{R}^{n}$ coincide avec le semi-groupe de la chaleur de l'espace $\mathbb{R}^{m+n}$. 
Une fonction $f$ sur $U \times V$ est dite séparément finement surharmonique si pour tout couple $(x, y) \in U \times V$, les fonctions $f(\cdot, y)$ et $f(x, \cdot)$ sont finement surharmoniques sur $U$ et $V$ respectivement.

D’après ce qui précède et le Théorème 1.1, on déduit qu'une fonction séparément finement surharmonique $\geq 0$ sur $U \times V$ est finement surharmonique.

Plus généralement, considérons deux opérateurs différentiel du second ordre $L^{m}$ et $L^{n}$ sur $\mathbb{R}^{m}$ et $\mathbb{R}^{n}$ respectivement définis par

$$
\begin{aligned}
L^{m} & =\sum_{1 \leq i<j \leq m} a_{i j}^{m} \frac{\partial^{2}}{\partial x_{i} \partial x_{j}}+\sum_{1 \leq i \leq m} b_{i}^{m} \frac{\partial}{\partial x_{i}}+c^{m}, \\
L^{n} & =\sum_{1 \leq i<j \leq n} a_{i j}^{n} \frac{\partial^{2}}{\partial x_{i} \partial x_{j}}+\sum_{1 \leq i \leq n} b_{i}^{n} \frac{\partial}{\partial x_{i}}+c^{n},
\end{aligned}
$$

où les coefficient $a_{i j}^{k}$ et $b_{i}^{k}, c^{k}, k=m, n$, sont des fonctions. On suppose qu'il existe un réel $\lambda \geq 1$ tel que les coefficients $a_{i j}^{k}$ et $b_{i}^{k}, k=m, n$, vérifient les conditions suivantes :

(1) $\forall x \in \mathbb{R}^{k}, \forall \xi \in \mathbb{R}^{k}, \sum a_{i j}^{k}(x) \xi_{i} \xi_{j} \geq(1 / \lambda)\|\xi\|^{2}$,

(2) $\forall x, x^{\prime} \in \mathbb{R}^{k}, \sum_{i j}\left|a_{i j}^{k}(x)-a_{i j}^{k}\left(x^{\prime}\right)\right|+\sum_{j}: \sum_{j}\left|b_{j}^{k}(x)-b_{j}^{k}\left(x^{\prime}\right)\right|+\left|c(x)-c\left(x^{\prime}\right)\right| \leq$ $\lambda\left\|x-x^{\prime}\right\|^{\alpha}$

(3) $\forall x \in \mathbb{R}^{k}, \sum_{i j}\left|a_{i j}^{k}(x)\right|+\sum_{j}: \sum_{j}\left|b_{j}^{k}(x)-b_{j}^{k}\left(x^{\prime}\right)\right|+|c(x)| \leq \lambda$.

À ces opérateurs sont associés des faisceaux de Brelot notés par $\mathscr{H}_{k}, k=1,2$ (voir [6]) : les $u \in \mathscr{H}_{k}(\Omega), \Omega$ ouvert de $\mathbb{R}^{n}$, sont les solutions de classe $\mathscr{C}^{2}$ sur $\Omega$ de l'équation $L^{k} u=0$. On sait aussi que $\mathscr{H}_{k}$ vérifie les propriétés d'unicité des potentiels de support ponctuels. On rappelle que d'après [6] l'effilement (au sens local) par rapport à $L^{k}$, $k=1,2$, coincide avec l'effilement classique, et de ce fait la topologie fine associée à $L^{k}$ coincide avec la topologie fine classique. On en déduit que la topologie produit des topologies fines de $\mathbb{R}^{m}$ et $\mathbb{R}^{k}$ est (strictement) moins fine que la topologie fine de $\mathbb{R}^{m+n}$ au sens de l'opérateur, noté par $L^{m+n}$ et défini par

$$
\begin{aligned}
L^{m+n}= & \sum_{1 \leq i<j \leq m} a_{i j}^{m} \frac{\partial^{2}}{\partial x_{i} \partial x_{j}}+\sum_{m+1 \leq i<j \leq m+n} a_{i j}^{n} \frac{\partial^{2}}{\partial x_{i} \partial x_{j}}+\sum_{1 \leq i \leq m} b_{i}^{m} \frac{\partial}{\partial x_{i}} \\
& +\sum_{m+1 \leq i \leq m+n} b_{i}^{n} \frac{\partial}{\partial x_{i}}+c^{m}+c^{n} .
\end{aligned}
$$

En utilisant les formes de Dirichlet, par exemple, on peut construire deux semigroupes $\left(P_{t}^{m}\right)$ et $\left(P_{t}^{n}\right)$ sur $\mathbb{R}^{m}$ et $\mathbb{R}^{n}$ respectivement et des diffusions $\left(X_{t}^{m}\right)$ et $\left(X_{t}^{n}\right)$ associés à ces semi-groupes. Les semi-groupes $\left(P_{t}^{m}\right)$ et $\left(P_{t}^{n}\right)$ sont absolument continus par rapport au mesures de Lebesgue sur $\mathbb{R}^{m}$ et $\mathbb{R}^{n}$ et on a, comme pour le semi-groupe du mouvement brownien,

$$
P_{t}^{m} \otimes P_{t}^{n}=P_{t}^{m+n}, \quad \forall t>0
$$

Ces observations permettent donc de montrer qu'une fonction séparément finement surharmonique $\geq 0$ au sens des opérateurs $L^{m}$ et $L^{n}$ sur un produit $U \times V$ de deux ouverts fins de $\mathbb{R}^{m}$ et $\mathbb{R}^{n}$ est finement surharmonique sur $U \times V$. 
REMERCIEMENTS. L'auteur tient à remercier l'arbitre pour les remarques et suggestions qu'il a bien voulu lui faire et qui ont conduit à la présente version de ce travail.

\section{BIBLIOGRAPHIE}

[1] R. M. Blumenthal and R. K. Getoor, Markov Processes and Potential Theory, Pure and Applied Mathematics, vol. 29, Academic Press, New York, 1968.

[2] R. Cairoli, Semi-groupes de transition et fonctions excessives, Séminaire de Probabilités (Univ. Strasbourg, Strasbourg, 1966/67), Vol. I, Lecture Notes in Mathematics, Springer-Verlag, Berlin, 1967, pp. 18-33 (French).

[3] C. Dellacherie and P.-A. Meyer, Probabilités et Potentiel. Chapitres XII-XVI [Probability and Potential. Chapters XII-XVI, Current Scientific and Industrial Topics, vol. 1417, Hermann, Paris, 1987 (French).

[4] M. El Kadiri, Fonctions séparément finement surharmoniques, à paraître dans Positivity.

[5] B. Fuglede, Localization in fine potential theory and uniform approximation by subharmonic functions, J. Funct. Anal. 49 (1982), no. 1, 57-72.

[6] R.-M. Hervé, Recherches axiomatiques sur la théorie des fonctions surharmoniques et $d u$ potentiel, Ann. Inst. Fourier (Grenoble) 12 (1962), 415-571 (French).

MOHAMEd El Kadiri : B.P. 726, SALÉ-TABriQUeT, SAlÉ, Morocco

E-mail address: elkadiri@fsr.ac.ma 


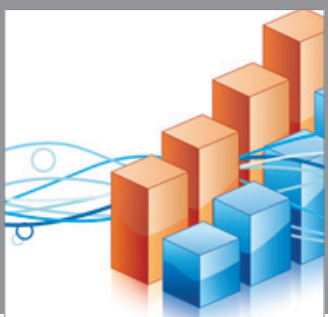

Advances in

Operations Research

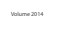

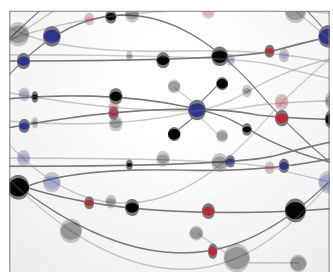

\section{The Scientific} World Journal
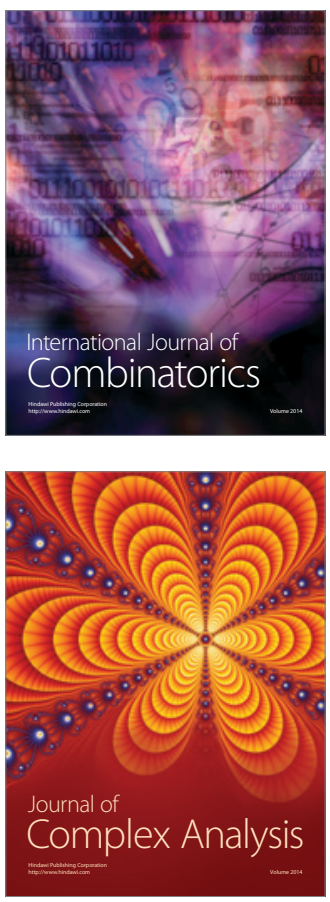

International Journal of

Mathematics and

Mathematical

Sciences
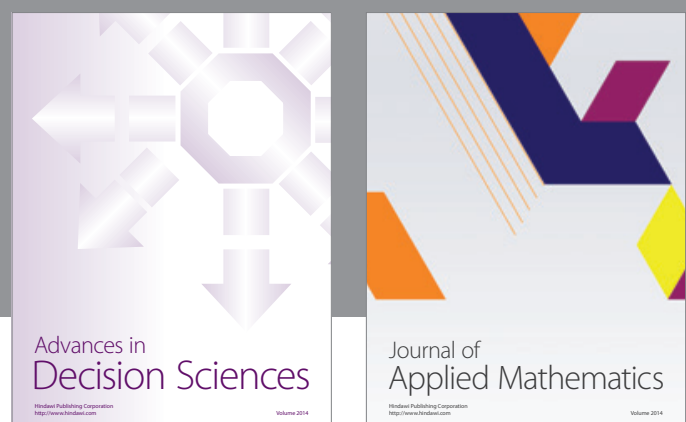

Journal of

Applied Mathematics
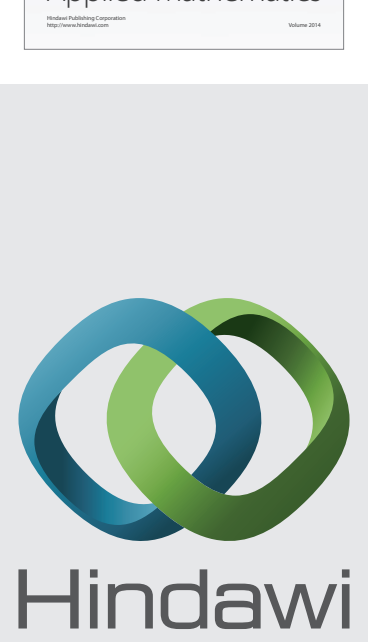

Submit your manuscripts at http://www.hindawi.com
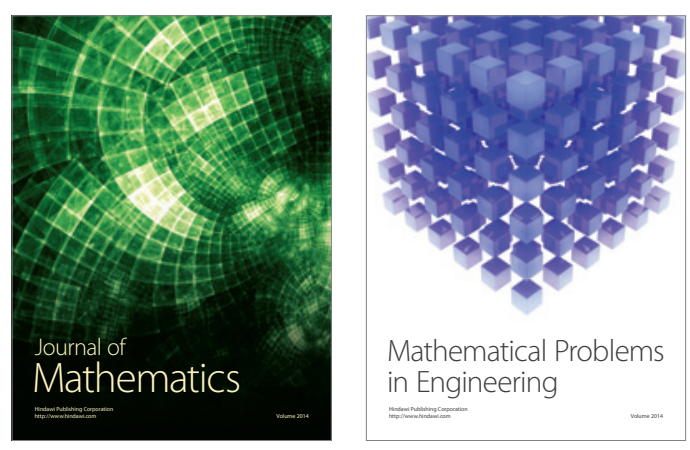

Mathematical Problems in Engineering
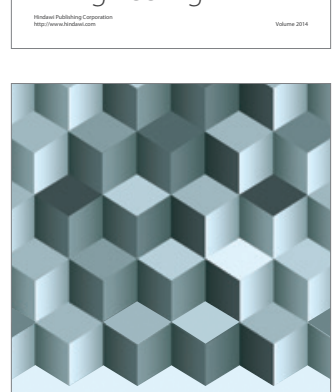

Journal of

Function Spaces
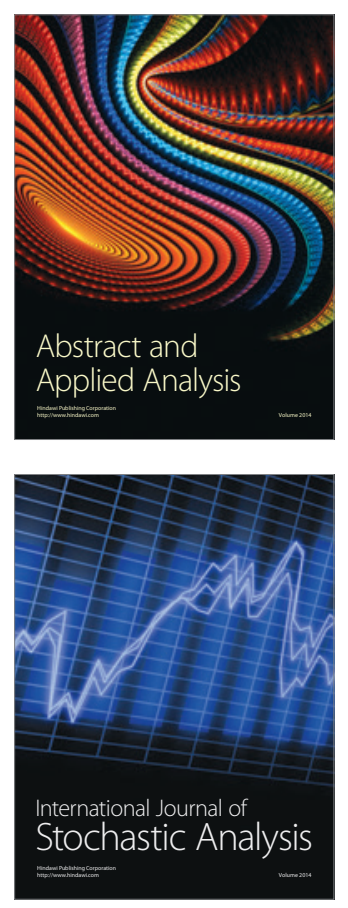

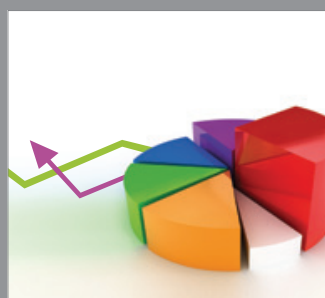

ournal of

Probability and Statistics

Promensencen
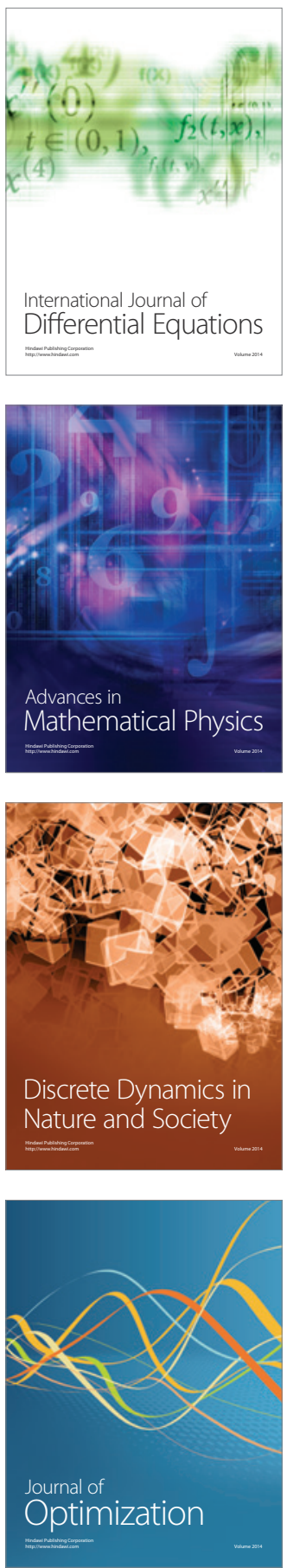